\title{
Inhibition of S100A9 expression by propofol in monocytes of rats with endotoxemia
}

\author{
ZHEN LI $^{1 *}$, GUODONG ZHAO ${ }^{1 *}$, GUOBIN ZHOU $^{1}$, YAN WANG $^{2}$, QING WANG ${ }^{1}$, \\ $\mathrm{JINQUAN} \mathrm{JI}^{1}$ and ZHIPENG WANG ${ }^{1}$ \\ ${ }^{1}$ Department of Anesthesiology, and ${ }^{2}$ Science and Education Department, Guangdong General Hospital, \\ Guangdong Academy of Medical Sciences, Guangzhou, Guangdong 510080, P.R. China
}

Received February 2, 2012; Accepted May 23, 2012

DOI: $10.3892 / \mathrm{mmr} .2012 .957$

\begin{abstract}
The inflammatory response is a non-specific autoimmune response. Monocytes are the most important effector cells in the systemic inflammatory response. In recent years, the function of the intravenous anesthetic, propofol, in the inhibition of the inflammatory response has attracted much attention. However, the specific signal transduction mechanism related to the anti-inflammatory effect of propofol remains unclear. In this study, monocyte protein expression in rats with endotoxemia was detected using proteomic techniques before and after propofol intervention. By two-dimensional electrophoresis and mass spectrometric identification, we found that S100A9 protein expression was significantly reduced after propofol treatment. In addition, we used western blot analysis to confirm the results of two-dimensional electrophoresis. The S100A9 protein, a member of the S100A calcium-binding protein family, is closely related to the occurrence and development of inflammation. The results of this study suggest that the anti-inflammatory effect of propofol may be related to the inhibition of S100A9 protein expression.
\end{abstract}

\section{Introduction}

Sepsis is a complex clinical syndrome generated by the interaction between the host and infectious pathogens and their toxic products, such as endotoxin and the host immune system, and inflammation and the coagulation response $(1,2)$. Severe sepsis may lead to multiple organ failure and is a common cause of mortality in ICU patients $(3,4)$. Endotoxin is a component of the outer membrane of the cell wall of Gram-negative bacteria. The chemical essence of endotoxin is lipopolysaccharide

Correspondence to: Professor Guobin Zhou, Department of Anesthesiology, Guangdong General Hospital, Guangdong Academy of Medical Sciences, No. 106 Zhongshan 2nd Road, Guangzhou, Guangdong 510080, P.R. China

E-mail: guobin_zhousy@163.com

*Contributed equally

Key words: propofol, lipopolysaccharide, endotoxemia, S100A9
(LPS) (5). LPS is the main triggering factor of sepsis and systemic inflammatory response syndrome (6). Monocytes are one of the important immune cells involved in inflammatory responses in the body $(7,8)$. Therefore, monocytes often serve as effector cells for the study of the inflammatory response.

Propofol, an intravenous anesthetic with a rapid onset of action and no accumulation during continuous infusion, is widely used in anesthesia induction, maintenance and sedative treatment of ICU patients, such as patients with sepsis (9). In recent years, the anti-inflammatory effects of propofol have gradually attracted more attention. Propofol can inhibit the release of the inflammatory cytokines, IL-1 $\beta$, IL- 6 and TNF- $\alpha(10,11)$; it is capable of removing oxygen free radicals and inhibiting oxidative damage generated by respiratory burst after neutrophil activation (12); it also inhibits the release of PF4 in the blood of rats with endotoxemia and partly alleviates hypercoagulability in endotoxemia (13). Although we have reached a consensus on the anti-inflammatory effects of propofol, its effects on monocyte protein expression remain unclear. In this study, comparative proteomic techniques were used to explore the impact of propofol on monocyte protein expression in rats with endotoxemia, in order to further clarify the molecular mechanisms of the anti-inflammatory action of propofol.

\section{Materials and methods}

Model building. The experiments were approved by the local ethics committee and performed in accordance with the Guidelines for the Care and Use of Animals of Guangdong General Hospital. In accordance with the guidelines of the International Association for the Study of Pain (14), all surgery was performed after the animals were anesthetized with urethane, so they did not feel pain or discomfort during the experiments, and the minimum possible pain or stress was imposed on the animals. In total, 18 male Sprague-Dawley rats weighing between 180 and $220 \mathrm{~g}$ anesthetized with urethane $(1.0 \mathrm{~g} / \mathrm{kg})$ intraperitoneally (i.p.) were divided into a control group, a LPS (Escherichia coli; Sigma, St. Louis, MO, USA) + intralipid (Sino-Swed Pharmaceutical Co., Ltd., Beijing, China) group and an LPS + propofol (FJ685; commercial name, Diprivan; AstraZeneca, London, UK) group. In the LPS + intralipid group, rats were infused with intralipid $\left(10 \mathrm{mg} \cdot \mathrm{kg}^{-1} \mathrm{~h}^{-1}\right)$ immediately following $10 \mathrm{mg} \cdot \mathrm{kg}^{-1} \mathrm{LPS}$ intravenously (i.v.), and in 
the LPS + propofol group rats were injected with LPS followed by propofol at $10 \mathrm{mg} \cdot \mathrm{kg}^{-1} \mathrm{~h}^{-1}$. In the control group, rats were injected the same amount of balanced saline. After $6 \mathrm{~h}, 3-4 \mathrm{ml}$ of blood from the carotid artery of each rat were collected into Eppendorf tubes and the rats were sacrificed thereafter.

Isolation of leukocytes from rat peripheral blood. In total, $3.0 \mathrm{ml}$ Histopaque 10831 (Sigma) were added to a $15-\mathrm{ml}$ centrifuge tube, then $3.0 \mathrm{ml}$ whole blood were carefully layered onto the Histopaque 10831 surface and centrifuged at $400 \mathrm{x} \mathrm{g}$ for $30 \mathrm{~min}$ at room temperature. After centrifugation, the upper layer to within 2-3 $\mathrm{mm}$ of the opaque interface contained the mononuclear cells. The upper layer was carefully aspirated with a Pasteur pipet and discarded. The opaque interface, containing the mononuclear cell band, was carefully transferred with a Pasteur pipet into a clean $15 \mathrm{ml}$ conical centrifuge tube. Isotonic PBS (10 ml) was then added to the mononuclear cells, the tube was mixed by gentle inversion several times and centrifuged at $250 \mathrm{x}$ g for $10 \mathrm{~min}$. The supernatant was aspirated and discarded. After resuspending the cell pellet with $0.5 \mathrm{ml}$ of isotonic PBS, an additional $4.5 \mathrm{ml}$ of isotonic PBS was added and the mixture was centrifuged at $250 \mathrm{xg}$ for $10 \mathrm{~min}$. The supernatant was aspirated and discarded. This step was repeated 2-3 times to remove any remaining Histopaque 10831 from the mononuclear cells. After the final wash, the cells were added to $300 \mu \mathrm{l}$ of lysis buffer consisting of $7 \mathrm{M}$ urea, $2 \mathrm{M}$ thiourea, 4\% CHAPS, $65 \mathrm{mM}$ DTT and 2\% Pharmalyte (pH 3-10; GE Healthcare, Piscataway, NJ, USA) by sonication on ice. The lysates were cleared by centrifugation at $12,000 \mathrm{rpm}$ for $30 \mathrm{~min}$ at $4^{\circ} \mathrm{C}$, twice. Subsequently, the protein concentration of the supernatants was determined by the modified Bradford method and the protein samples were stored at $-80^{\circ} \mathrm{C}$.

Two-dimensional (2D) electrophoresis. An Immobiline DryStrip (pH 3.0-10.0, length $24 \mathrm{~cm}$, GE Healthcare) was rehydrated with $1,500 \mu \mathrm{g}$ protein in $450 \mathrm{ml}$ rehydration buffer containing $7 \mathrm{M}$ urea, $2 \mathrm{M}$ thiourea, 4\% CHAPS, $65 \mathrm{mM}$ DTT, $20 \mathrm{mM}$ Trizma base, $1 \%$ IPG buffer and $0.002 \%$ bromophenol blue for $14 \mathrm{~h}$ at room temperature. Isoelectric focusing (IEF) was performed using the Ettan IPGphor 3 IEF System (GE Healthcare) for a total of $70 \mathrm{kVh}$. The strip was then subjected to two-step equilibration in a buffer containing $6 \mathrm{M}$ urea, 20\% glycerol, $2 \%$ SDS and $50 \mathrm{mM}$ Tris- $\mathrm{HCl}(\mathrm{pH} 8.8)$ with $2 \% \mathrm{w} / \mathrm{v}$ DTT for the first step, and $2.5 \% \mathrm{w} / \mathrm{v}$ iodoacetamide for the second step. The second-dimension SDS-PAGE $\left(12 \% \mathrm{~T}, 260 \times 200 \times 1.5 \mathrm{~mm}^{3}\right)$ was carried out using a Ettan DALTsix Large Vertical system (Amersham Pharmacia Biotech, Piscataway, NJ, USA) according to the following procedures: $45 \mathrm{~min}$ at a constant power of 5 watt followed by 20 watt per gel until the bromophenol blue front reached the bottom of the gel. Subsequently, the gels were stained with $0.12 \%$ w/v Coomassie Brilliant Blue G250. Each group was run in triplicate to minimize run-to-run variation. The Coomassie Blue-stained protein 2D gels were scanned using an Amersham Biosciences ImageScanner and analyzed using a DeCyder software package (GE Healthcare).

In-gel digestion. Protein spots were excised from the gel with an operating knife blade, destained twice with $30 \mathrm{mM}$ potassium ferricyanide and $100 \mathrm{mM}$ sodium thiosulfate $(1: 1 \mathrm{v} / \mathrm{v})$ and then equilibrated in $100 \mathrm{mM} \mathrm{NH}_{4} \mathrm{HCO}_{3}$ to $\mathrm{pH}$ 8.0. After dehydrating with acetonitrile (ACN) and drying in nitrogen at $37^{\circ} \mathrm{C}$ for $20 \mathrm{~min}$, the gel pieces were rehydrated in $10 \mu \mathrm{l}$ trypsin solution $\left(12.5 \mathrm{ng} / \mu \mathrm{l}\right.$ in $\left.50 \mathrm{mM} \mathrm{NH} \mathrm{NCO}_{3}\right)$ at $4^{\circ} \mathrm{C}$ for $30 \mathrm{~min}$ and incubated at $37^{\circ} \mathrm{C}$ overnight. Peptides were extracted twice using $0.1 \%$ trifluoroacetic acid (TFA) in $60 \%$ CAN and dried with the RCT60 (Jouan S.A., Jouan, France).

Matrix-assisted laser desorption ionization time-of-flight mass spectrometry (MALDI-TOF-MS) identification. The peptide mixtures were solubilized with $0.1 \%$ TFA and desalted with C18 ZipTip (Millipore, USA). The peptide was then eluted by saturated a-cyano-4-hydroxy-trans-cinnamic (CHCA) solution in $0.1 \% \mathrm{TFA} / 60 \%$ acetonitrile as the matrix and analyzed using a 4800 MALDI TOF/TOF analyzer (Applied Biosystems, USA). Mass spectra were internally calibrated with angiotensin I (Mr: 1296.6853).

Protein identification and database searching. Protein identification using peptide mass fingerprinting (PMF) and peptide sequence tag (PST) was performed using the Mascot search engine (http://www.matrixscience.com, MatrixSicence Ltd., London, UK) against the SwissProt protein database. The errors in peptide masses were in the range of $50 \mathrm{ppm}$. One missed tryptic cleavage site per peptide was allowed during the search. Proteins matching more than 4 peptides and with a MASCOT score higher than 64 were considered significant $(\mathrm{P}<0.05)$. Carboamidomethylation of cysteine was selected as the fixed modification, and oxidation of methionine as the variable modification. Protein identification results were filtered with GPS software.

Western blot analysis. In order to verify the 2D electrophoresis protein expression data, another 36 Sprague-Dawley rats were divided into 6 groups: the control, intralipid, propofol, LPS, propofol + LPS and intralipid + LPS groups. In the intralipid and propofol group, rats were infused with intralipid or propofol at $10 \mathrm{mg} \cdot \mathrm{kg}^{-1} \mathrm{~h}^{-1}$. The LPS group was injected with LPS followed by the same amount of balanced saline and the other 3 groups were treated the same as above. S100A9 in the mononuclear cells of the rats was detected by western blot analysis. The mononuclear cells of the rats were lysed on ice in $300 \mu \mathrm{l}$ cell lysis buffer [1X PBS, 1\% NP40, 0.1\% sodium dodecyl sulfate (SDS), $5 \mathrm{mM}$ EDTA, $0.5 \%$ sodium deoxycholate and $1 \mathrm{mM}$ sodium orthovanadate] with protease inhibitors. Protein concentration was determined by the modified Bradford method. Equal amounts of protein were separated electrophoretically on $12 \%$ SDS/polyacrylamide gels and transferred onto polyvinylidene difluoride membranes (PVDF) (Amersham Pharmacia Biotech). For S100A9 detection, the membrane was probed with anti-S100A9 rabbit polyclonal antibody (1:1000; Abcam) and horseradish peroxidase-conjugated anti-rabbit immunoglobulin G (1:2000; Cell Signaling Technology, Danvers, MA, USA). The immunoreactive bands were visualized on a Kodak 2000M camera system (Eastman Kodak, Rochester, NY, USA) according to the manufacturer's instructions.

Statistical analysis. All the data were examined for normal distribution prior to statistical analysis and the statistical 
analysis was carried out using SPSS 13.0. All data were expressed as the means \pm SD. Multiple groups were compared using analysis of variance (ANOVA) followed by the StudentNewman-Keuls post-hoc procedure. $\mathrm{P}<0.05$ was considered to indicate statistically significant differences.

\section{Results}

Quantitative comparison and identification of protein spots on $2 \mathrm{D}$ gels. To determine the change in the monocyte protein profile in response to LPS and propofol, gel-based comparative proteomic analysis was performed. Thirteen protein spots were found to be significantly altered (data not shown). One of them was S100A9 identified by MALDI-TOF MS and by a subsequent comparative sequence search in the Mascot database (Fig. 1).

Verification of downregulation of S100A9 in the LPS + propofol group by western blot analysis in endotoxemic rats. As gel-based proteomic analysis showed that the expression of S100A9 decreased markedly in the LPS + propofol group, S100A9 was elected for further investigation. The corresponding differential expression patterns identified by 2D electrophoresis and the MALDI-TOF mass spectra are shown in Fig. 2. S100A9 is a 13,200 molecular weight pro-inflammatory protein expressed abundantly in the cytosol of monocytes and neutrophils (1). To more rigorously study the effect of propofol on the expression of S100A9 in the monocytes, 36 rats were divided into 6 groups: control group, intralipid group, propofol group, LPS group, LPS + propofol group and LPS + intralipid group. S100A9 expression in the monocytes of each group was determined by western blot analysis. As shown in Fig. 3A, the amount of S100A9 in monocytes showed no statistical difference in the control, intralipid, propofol and LPS + propofol groups $(\mathrm{P}>0.05)$. However, in the monocytes of the LPS and LPS + intralipid group, the expression of S100A9 increased markedly in accordance with our 2D gels results. These results indicate that propofol is capable of inhibiting the expression of S100A9 in the monocytes of endotoxemic rats. Total $\beta$-actin loaded on SDS-PAGE was used as the internal control (Fig. 3B).

\section{Discussion}

The anti-inflammatory effect of the intravenous anesthetic, propofol, has drawn much attention (10-12). It has previously been reported that propofol is capable of inhibiting the release of inflammatory cytokines, such as IL-1 $\beta$, IL-6 and TNF- $\alpha$ (12); however, the mechanisms by which propofol inhibits the secretion of inflammatory cytokines and the specific signal transduction mechanisms remain unclear. This also limits the clinical application of the anti-inflammatory effect of propofol. Monocytes are the most important effector cells in the systemic inflammatory response (7). Therefore, a rat model of endotoxemia by LPS was established and 2D electrophoresis and proteomic techniques were used to detect monocyte protein expression in serum to find propofol-related inflammatory proteins. By mass spectrometry, it was found that the inflammation-associated protein, S100A9, was significantly reduced in the LPS + propofol treatment group.
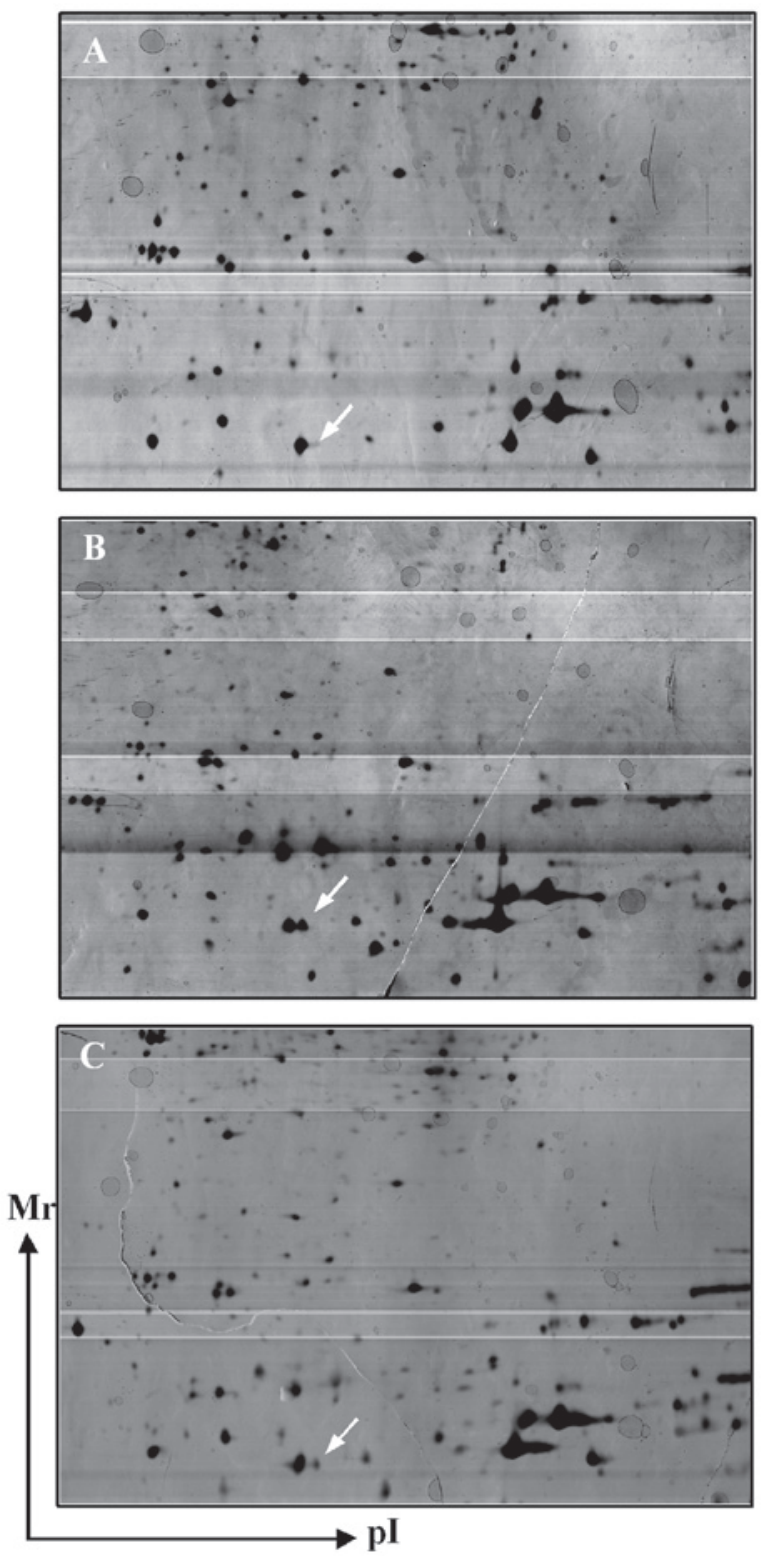

Figure 1. Representative Two-dimensional gels. Control group (upper panel), lipopolysaccharide (LPS) + intralipid group (middle panel) and LPS + propofol group (lower). The protein spot was successfully identified by matrix-assisted laser desorption ionization time-of-flight mass spectrometry (MALDI-TOF MS).

S100A9 is a key member of the S100 calcium-binding protein family. Its relative molecular weight is $13.2 \mathrm{kDa}(16)$. S100A9 is mainly expressed in human and rat monocyte/macrophage cell lines, neutrophils and keratinocytes under certain pathological conditions $(16,17)$. Previous studies have indicated that S100A9 levels are significantly increased in inflammatory states: after inflammation is induced by carrageenan in rats, S100A9 concentration is increased in exudates, and it plays a major role in chronic inflammation by stimulating the proliferation of fibroblasts (18). In the late stage of endotoxininduced uveitis, S100A9 may clear inflammation in cells (17). It is also closely related to systemic lupus erythematosus, giant cell arteritis, multiple sclerosis and many other immune inflammations (19). Studies have suggested that S100A9 is a specifically overexpressed marker in asthma (20). In the initial 
A

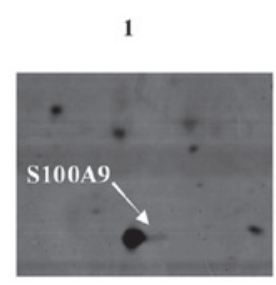

2

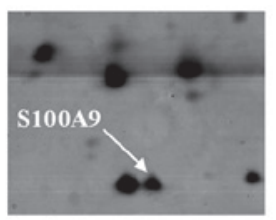

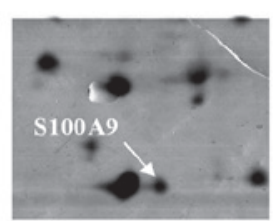

B

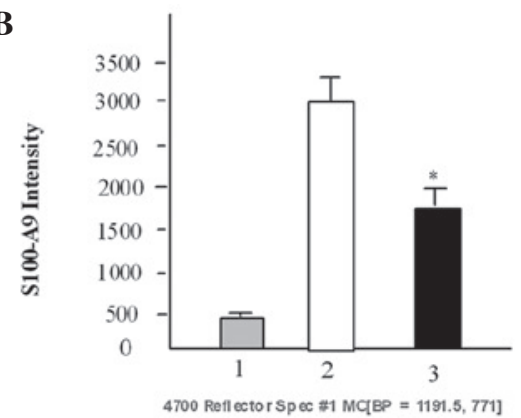

C

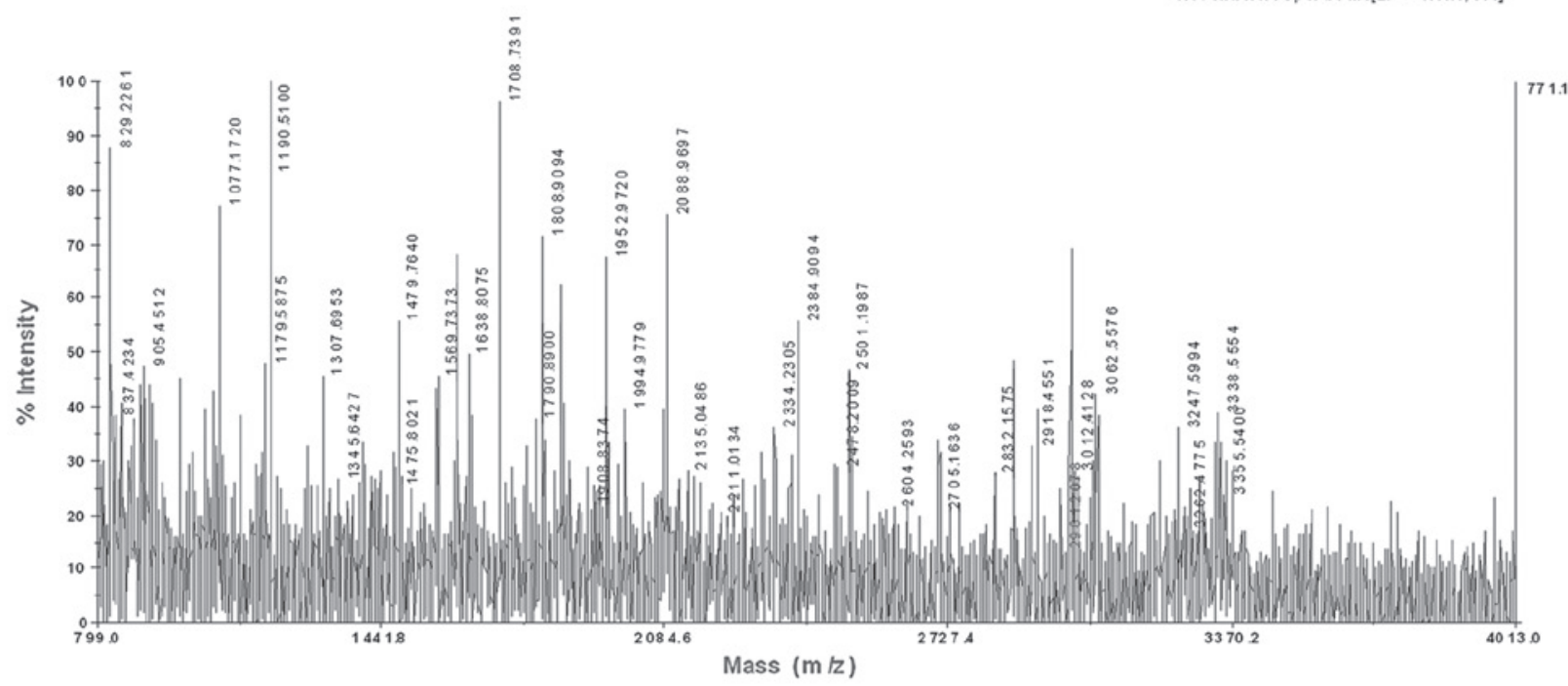

Figure 2. Identification of S100A9 downregulation in the lipopolysaccharide (LPS) + propofol group. (A) Gels in the control (1), LPS + intralipid (2), and LPS + propofol (3) groups arfe enlarged to show the high expression of S100A9 in the 3 groups. (B) The densitometric analysis of each protein was calculated from 9 different gels using PDQuest software. Each bar represents the mean \pm SD of intensity. The expression of S100A9 in the LPS + propofol group decreased markedly compared with the LPS + intralipid group ( $\left.{ }^{*} \mathrm{p}<0.05\right)$ and the control group ( $\left.{ }^{*} \mathrm{p}<0.05\right)$. (C) Mass spectometry of in-gel trypsin digests of the protein and analysis of the depicted peptide spectrum resulted in the identification of S100A9.

A

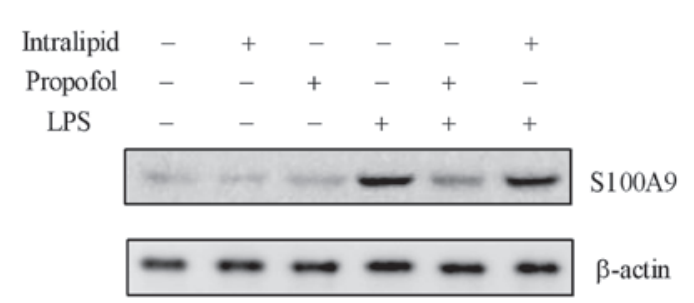

$\mathbf{B}$

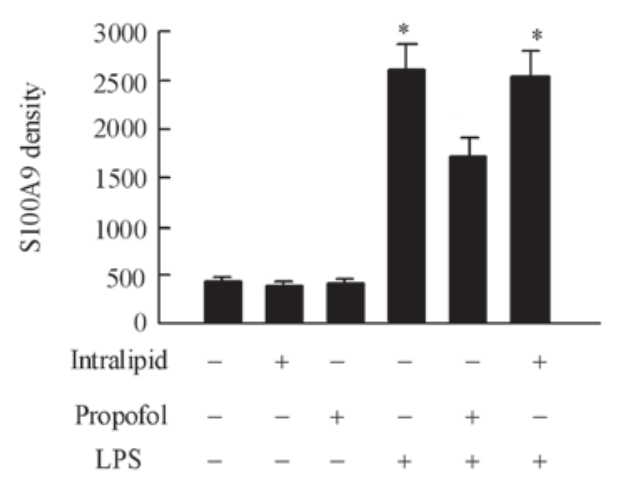

Figure 3. Confirmation of the expression of S100A9 in monocytes with western blot analysis. (A) Monocyte lysis samples from the individual rats were separated on $12 \%$ SDS-PAGE gels and transferred to PVDF membranes for western blot analysis. (B) Gray intensity analysis of the western blot results of the 6 groups. Each bar represents the mean $\pm \mathrm{SD}\left({ }^{*} \mathrm{p}<0.05, \mathrm{n}=6\right.$, compared with the other 4 groups). stage of inflammation, S100A9-positive cells express higher levels of CD11b. Studies have shown that the lack of S100A9 in cells in vitro reduces the response of white blood cells to chemical stimuli (21). A S100A9 gene knockout mouse model of pancreatitis showed that leukocyte infiltration was reduced in lung tissue and pancreatic tissue in mice, and serum amylase levels were also decreased (22). These findings have prompted the theory that S100A9 is closely related to the inflammatory response.

Therefore, in the present study, we chose the S100A9 protein for subsequent validation and research. In addition, we selected 36 Sprague-Dawley rats to give endotoxin, propofol or intralipid for stimulation and used western blot analysis to detect S100A9 protein expression in monocytes. The results showed that after LPS stimulation, S100A9 expression in monocytes was significantly increased, which to a certain extent, indicated that S100A9 is a marker of inflammatory response; following propofol treatment, S100A9 expression was significantly reduced, indicating that propofol can significantly inhibit the expression of the inflammatory protein, S100A9. Intralipid is a solvent of propofol. Certain studies have suggested that intralipid has anti-inflammatory effects and can inhibit the release of inflammatory cytokines (23); however, our results showed that the administration of intralipid after LPS stimulation did not inhibit the expression of S100A9 protein. 
Mitogen-activated protein kinase (MAPK) pathways are one of the important signal transduction systems in organisms. They are capable of mediating the signal responses of cells to a variety of external stresses. p38MAPK, ERK and JNK are 3 relatively important signaling pathways (24). These MAPK pathways may be activated by a variety of inflammatory stimuli, such as LPS and play an important role in regulating the occurrence and development of inflammation. Previous studies have suggested that the effects of S100A9 and MAPK signal transduction pathways are very similar: S100A9, as a reliable marker of inflammation in macrophages, is capable of specifically activating JNK and ERK pathways through TLR4, and at the gene and protein levels, can induce nitric oxide synthase expression (25). Under pathological conditions, S100A9 can be specifically phosphorylated by p38MAPK kinase, decreasing the aggregating ability of cell membranes, thus promoting the travelling of inflammatory cells. S100A9 can also have a reverse effect on p38 kinase, inhibiting cell migration (26). The study of Tang et al (27) showed that propofol plays an anti-inflammatory role by inhibiting p38 phosphorylation. S100A9 not only affects inflammatory responses, but can also regulate tumor cell proliferation, metabolism and migration. It plays a promoting role in the physiological and pathological process of tumors. El-Rifai et al (28) found that the S100A9 gene was overexpressed in gastric cancer epithelial cells using serial analysis of gene expression; the immunohistochemical results showed that S100A9 was overexpressed in the macrophages and leukocyte-infiltrated tissue edge in colorectal cancer (29). Propofol has also been shown to have anti-tumor properties (30). It is speculated from the literature that the antiinflammatory and anti-tumor properties of propofol correlate with the S100A9 protein and the MAPK signaling pathway.

In conclusion, the results from this study suggest that propofol inhibits the expression of the S100A9 protein; however, the upstream and downstream acting elements in the inhibition of S100A9 protein expression are not yet clear. In a subsequent experiment, we will investigate the correlation between S100A9 and the MAPK pathway and observe whether there is an interaction between them and further improve the molecular basis of the anti-inflammatory effects of propofol.

\section{Acknowledgements}

This study was supported by the Guangdong Science and Technology Plan (2006B36007015).

\section{References}

1. Brunkhorst FM and Reinhart K: Diagnosis and causal treatment of sepsis. Internist (Berl) 50: 810-816, 2009.

2. Sharma S and Kumar A: Septic shock, multiple organ failure, and acute respiratory distress syndrome. Curr Opin Pulm Med 9: 199-209, 2003.

3. Schneider J and Muleta M: Septic shock. Ethiop Med J 41: 89-104, 2003.

4. Tabbutt S: Heart failure in pediatric septic shock: utilizing inotropic support. Crit Care Med 29 (Suppl 10): S231-S236, 2001.

5. Heine H, Rietschel ET and Ulmer AJ: The biology of endotoxin. Mol Biotechnol 19: 279-296, 2001.

6. Leslie DB, Vietzen PS, Lazaron V, et al: Comparison of endotoxin antagonism of linear and cyclized peptides derived from limulus anti-lipopolysaccharide factor. Surg Infect (Larchmt) 7: 45-52, 2006.
7. Wu J F, Ma J, Chen J, et al: Changes of monocyte human leukocyte antigen-DR expression as a reliable predictor of mortality in severe sepsis. Crit Care 15: R220, 2011.

8. Monneret G, Lepape A, Voirin N, et al: Persisting low monocyte human leukocyte antigen-DR expression predicts mortality in septic shock. Intensive Care Med 32: 1175-1183, 2006.

9. Sztark F and Lagneau F: Agents for sedation and analgesia in the intensive care unit. Ann Fr Anesth Reanim 27: 560-566, 2008 (In French).

10. Lee C J, Subeq Y M, Lee R P, et al: Low-dose propofol ameliorates haemorrhagic shock-induced organ damage in conscious rats. Clin Exp Pharmacol Physiol 35: 766-774, 2008.

11. Chu C H, David L D, Hsu Y H, et al: Propofol exerts protective effects on the acute lung injury induced by endotoxin in rats. Pulm Pharmacol Ther 20: 503-512, 2007.

12. Tsao C M, Ho S T, Chen A, et al: Propofol ameliorates liver dysfunction and inhibits aortic superoxide level in conscious rats with endotoxic shock. Eur J Pharmacol 477: 183-193, 2003.

13. Tang J, Sun Y, Wu W K, et al: Propofol lowers serum PF4 level and partially corrects hypercoagulopathy in endotoxemic rats. Biochim Biophys Acta 1804: 1895-1901, 2010.

14. Zimmermann M: Ethical guidelines for investigations of experimental pain in conscious animals. Pain 16: 109-110, 1983.

15. Simard JC, Girard D and Tessier PA: Induction of neutrophil degranulation by S100A9 via a MAPK-dependent mechanism. J Leukoc Biol 87: 905-914, 2010.

16. Hessian PA, Edgeworth J and Hogg N: MRP-8 and MRP-14, two abundant $\mathrm{Ca}(2+)$-binding proteins of neutrophils and monocytes. J Leukoc Biol 53: 197-204, 1993

17. Chi ZL, Hayasaka Y, Zhang XY, et al: S100A9-positive granulocytes and monocytes in lipopolysaccharide-induced anterior ocular inflammation. Exp Eye Res 84: 254-265, 2007.

18. Shibata F, Miyama K, Shinoda F, et al: Fibroblast growthstimulating activity of S100A9 (MRP-14). Eur J Biochem 271: 2137-2143, 2004.

19. Gebhardt C, Nemeth J, Angel P, et al: S100A8 and S100A9 in inflammation and cancer. Biochem Pharmacol 72: 1622-1631, 2006.

20. Yin LM, Jiang GH, Wang Y, et al: Use of serial analysis of gene expression to reveal the specific regulation of gene expression profile in asthmatic rats treated by acupuncture. J Biomed Sci 16: 46, 2009.

21. Manitz MP, Horst B, Seeliger S, et al: Loss of S100A9 (MRP14) results in reduced interleukin-8-induced CD11b surface expression, a polarized microfilament system, and diminished responsiveness to chemoattractants in vitro. Mol Cell Biol 23: 1034-1043, 2003

22. Schnekenburger J, Schick V, Kruger B, et al: The calcium binding protein S100A9 is essential for pancreatic leukocyte infiltration and induces disruption of cell-cell contacts. J Cell Physiol 216: 558-567, 2008.

23. Yang YH, Toh ML, Clyne CD, et al: Annexin 1 negatively regulates IL-6 expression via effects on p38 MAPK and MAPK phosphatase-1. J Immunol 177: 8148-8153, 2006.

24. Kaminska B: MAPK signalling pathways as molecular targets for anti-inflammatory therapy--from molecular mechanisms to therapeutic benefits. Biochim Biophys Acta 1754: 253-262, 2005.

25. Pouliot P, Plante I, Raquil MA, et al: Myeloid-related proteins rapidly modulate macrophage nitric oxide production during innate immune response. J Immunol 181: 3595-3601, 2008.

26. Hiratsuka S, Watanabe A, Aburatani H, et al: Tumour-mediated upregulation of chemoattractants and recruitment of myeloid cells predetermines lung metastasis. Nat Cell Biol 8: 1369-1375, 2006.

27. Tang J, Chen X, Tu W, et al: Propofol inhibits the activation of p38 through up-regulating the expression of annexin A1 to exert its anti-inflammation effect. PLoS One 6: e27890, 2011.

28. El-Rifai W, Moskaluk CA, Abdrabbo MK, et al: Gastric cancers overexpress S100A calcium-binding proteins. Cancer Res 62: 6823-6826, 2002.

29. Stulik J, Osterreicher J, Koupilova K, et al: The analysis of S100A9 and S100A8 expression in matched sets of macroscopically normal colon mucosa and colorectal carcinoma: the S100A9 and S100A8 positive cells underlie and invade tumor mass. Electrophoresis 20: 1047-1054, 1999.

30. Tsuchiya M, Asada A, Arita K, et al: Induction and mechanism of apoptotic cell death by propofol in HL-60 cells. Acta Anaesthesiol Scand 46: 1068-1074, 2002. 